\title{
SOME CLASSES OF ANALYTIC FUNCTIONS RELATED WITH BAZILEVIČ FUNCTIONS
}

\author{
KHALIDA INAYAT NOOR
}

\begin{abstract}
A certain class $M_{k}(\alpha, \beta)$ of analytic functions is introduced and it is shown that $M_{2}(\alpha, \beta)$ is contained in the class of Bazilevic functions. Some other properties of $M_{k}(\alpha, \beta)$ are also derived.
\end{abstract}

\section{Introduction}

Let $A$ denote the class of functions $f: f(z)=z+\sum_{n=2}^{\infty} a_{n} z^{n}$ which are analytic in the unit disc $E=\{z:|z|<1\}$. By $S, K, S^{*}$ and $C$, we denote the subclasses of $A$ which are respectively univalent, close-to-convex, starlike (with respect to the origin) and convex in $E$.

Let $P_{k}$ be the class of analytic function $p$ defined in $E$ and with representation

$$
p(z)=\frac{1}{2} \int_{-\pi}^{\pi} \frac{1+z e^{-i t}}{1-z e^{-i t}} d \mu(t)
$$

where $\mu(t)$ is a function with bounded variation on $[-\pi, \pi]$ and it satisfies the condicions

$$
\int_{-\pi}^{\pi} d \mu(t)=2, \quad \int_{-\pi}^{\pi}|d \mu(t)| \leq k .
$$

We note that $k \geq 2$ and $P_{2}=P$ is the class of analytic functions with positive real part in $E$ with $p(0)=1$. The class $P_{k}$ was introduced in [3]. From the integral representation (1.1) it is immediately clear that $p \in P_{k}$ if and only if there are analytic function $p_{1}, p_{2} \in$ $P$ such that

$$
p(z)=\left(\frac{k}{4}+\frac{1}{2}\right) p_{1}(z)-\left(\frac{k}{4}-\frac{1}{2}\right) p_{2}(z) .
$$

We defined the Hadamard product or Convolution of two analytic functions $f(z)=$ $\sum_{n=0}^{\infty} a_{n} z^{n+1}$ and $g(z)=\sum_{n=0}^{\infty} b_{n} z^{n+1}$ as

$$
(f * g)(z)=\sum_{n=0}^{\infty} a_{n} b_{n} z^{n+1}, \quad z \in E .
$$

Received September 2, 1996.

1991 Mathematics Subject Classification. Primary 30C45.

Key words and phrases. Bazilevič functions, bounded boundary rotation, Hadamard product, radius of convexity. 
We now define the following.

Definition 1.1. A function $f \in A$ is said to be belong to the class $M_{k}\left(\alpha, \beta^{\prime}\right)$ if and only if it satisties the property

$J(\alpha, \beta ; f(z), g(z))=\left\{\frac{z f^{\prime}(z)}{f^{1-\beta}(z) g^{\beta}(z)}+\alpha\left(1+\frac{z f^{\prime \prime}(z)}{f^{\prime}(z)}\right)-\alpha(1-\beta) \frac{z f^{\prime}(z)}{f(z)}-\alpha \beta \frac{z g^{\prime}(z)}{g(z)}\right\} \in P_{k}$, for some real $\alpha, \beta(\beta \geq 0), g \in A$ and $z \in E$.

\section{Special Cases}

(i) For $k=2, \alpha=0$ and $g \in S^{*}$, we obtain the sell-known class $B(\beta) \subset S$ of Bazilevič functions of type $\beta$.

(ii) When $\beta=0$, the class $M_{k}(\alpha, 0)$ consists of functions with bounded Mocanu variation, see [1] and $M_{2}(\alpha, 0)$ is the class of $\alpha$-starlike functions which are known to be starlike univalent in $E$.

(iii) $M_{k}(1,0)=V_{k}$ is the class of functions with bounded boundary rotation and $M_{2}(1,0)=C$.

(iv) $M_{k}(0,0)$ is the class $R_{k}$ of functions with bounded radius rotation and $M_{2}(0,0)=$ $S^{*}$.

(v) For $\alpha=0, \beta=1$ and $g \in R_{k}$, we have $M_{2}(0,1)=T_{k}$ which has been introduced and studied in [2]. Also for $g \in S^{*}$, we note that $M_{2}(0,1)=K$.

\section{Preliminary Results}

Lemma 2.1. Let $p$ be analytic in $E$ and $p(0)=1$. Then, for $\alpha \geq 0, z \in E$, $\left(p+\alpha \frac{z p^{\prime}}{p}\right) \in P_{k}$ implies $p \in P_{k}$ in $E$.

The proof follows directly from the result, proved in [1], that functions with bounded Mocanu variation are in $R_{k}$.

From the Herglotz representation (1.1) for $k=2$, we have the following result.

Lemma 2.2. If $p$ is analytic in $E, p(0)=1$ and $\operatorname{Rep} p(z)>\frac{1}{2}, z \in E$, then for any function $F$, analytic in $E$, the function $p * F$ takes values in the convex hull of the image of $E$ under $F$.

\section{Main Results}

Theorem 3.1. For $\alpha \geq 0, M_{k}(\alpha, \beta) \subset M_{k}(0, \beta)$.

Proof. Let $f \in M_{k}(\alpha, \beta)$. We define

$$
\frac{z f^{\prime}(z)}{f^{1-\beta}(z) g^{\beta}(z)}=H(z)
$$


We see that $H(0)=1$ and $H$ is analytic in $E$. Logarithmic differentiation of (3.1) gives us

$$
\alpha\left(1+\frac{z f^{\prime \prime}(z)}{f^{\prime}(z)}\right)-\alpha(1-\beta) \frac{z f^{\prime}(z)}{f(z)}-\alpha \beta \frac{z g^{\prime}(z)}{g(z)}=\alpha z \frac{H^{\prime}(z)}{H(z)},
$$

and so

$$
J(\alpha, \beta ; f, g)=H(z)+\alpha \frac{z H^{\prime}(z)}{H(z)} .
$$

Since $f \in M_{k}(\alpha, \beta)$, it follows that $\left(H+\alpha \frac{z H^{\prime}}{H}\right) \in P_{k}$ and using Lemma 2.1, we conclude that $H \in P_{k}$. Consequently $f \in M_{k}(0, \beta)$.

Corollary 3.1. Let $g \in S^{*}$ and $k=2$. Then, for $\alpha \geq 0$,

$$
M_{2}(\alpha, \beta) \subset S
$$

since in this case $\frac{z f^{\prime}(z)}{f^{1-\beta}(z) g^{\beta}(z)} \in P, g \in S^{*}$ implies $f$ is Bazilevič and hence univalent.

Corollary 3.2. For $\alpha \geq 0, M_{k}(\alpha, 0) \subset M_{k}(0,0)$. That is a function with bounded Mocanu variation is of bounded radius rotation. With $k=2$ we also deduce that $\alpha$-starlike functions are starlike.

Corollary 3.3. Let $g \in S^{*}$ and $k=2, \beta=1$. Then $M_{2}(\alpha, 1) \subset M_{2}(0,1)=K \subset S$. That is $f \in M_{2}(\alpha, 1)$ is a close-to-convex univalent function.

In the opposite direction we prove the following.

Theorem 3.2. Let $f \in M_{k}(0, \beta)$. Then $f \in M_{k}(\alpha, \beta)$ for $|z|<r_{\alpha}$ where

$$
r_{\alpha}=\frac{1}{2 \alpha+\sqrt{4 \alpha^{2}-2 \alpha+1}} .
$$

Proof. With $\frac{z f^{\prime}(z)}{f^{1-\beta}(z) g^{\beta}(z)}=H(z) \in P_{k}$, we have

$$
J(\alpha, \beta ; f, g)=H(z)+\alpha \frac{z H^{\prime}(z)}{H(z)} .
$$

Since $H \in P_{k}$, we use (1.3) to write

$$
\begin{gathered}
H(z)=\left(\frac{k}{4}+\frac{1}{2}\right) h_{1}(z)-\left(\frac{k}{4}-\frac{1}{2}\right) h_{2}(z), \quad h_{1}, h_{2} \in P . \\
\text { Let } \phi_{\alpha}(z)=(1-\alpha) \frac{z}{1-z}+\alpha \frac{z}{(1-z)^{2}}=z+\sum_{n=2}^{\infty}\left[1+(n-1) \alpha z^{n}\right] .
\end{gathered}
$$

$\phi_{\alpha}$ is convex for $|z|<r_{\alpha}$ and this is sharp and so, for $|z|<r_{\alpha}$, $\operatorname{Re} \frac{\phi_{\alpha}(z)}{z}>\frac{1}{2}$. Thus

$$
\left(H * \frac{\phi_{\alpha}}{z}\right)=H+\alpha \frac{z H^{\prime}}{H}=\left(\frac{k}{4}+\frac{1}{2}\right)\left(h_{1} * \frac{\phi_{\alpha}}{z}\right)-\left(\frac{k}{4}-\frac{1}{2}\right)\left(h_{2} * \frac{\phi_{\alpha}}{z}\right) .
$$


Using Lemma 2.2, we see that $\left(h_{i} * \frac{\phi_{\alpha}}{z}\right) \in P$ for $|z|<r_{\alpha}, i=1,2$ and hence $\left(H+\alpha \frac{z H^{\prime}}{H}\right) \in P$ for $|z|<r_{\alpha}$. Consequently, from (3.3), it follows that $f \in M_{k}(\alpha, \beta)$ for $|z|<r_{\alpha}$ where $r_{\alpha}$ is given by (3.2).

Corollary 3.4. Let $f \in M_{k}(0,0)$. Then $f \in M_{k}(\alpha, 0)$ for $|z|<r_{\alpha}$, where $r_{\alpha}$ is given by (3.2). That is $f \in R_{k}$ implies $f$ is of bounded Mocanu variation for $|z|<r_{\alpha}$.

Corollary 3.5. In Corollary 3.4 we take $\alpha=1$. Then it follows that $f \in R_{k}$ implies $f \in V_{k}$ for $|z|<\frac{1}{2+\sqrt{3}}=2-\sqrt{3}$ and $k=2$ gives us the radius of convexity for starlike functions.

\section{References}

[1] H. B. Coonce and M. R. Ziegler, "Functions with bounded Mocanu variation," Rev. Roum. Math. Pures et Appl., 19 (1974), 1093-1104.

[2] K. I. Noor, "On a generalization of close-to-convexity," Int. J. Math. and Math. Sci., 6 (1983), 327-334.

[3] B. Pinchuk, "Functions with bounded boundary rotation," I. J. Math., 10 (1971), 7-16.

Mathematics Department, College of Science, King Saud University, P.O. Box 2455, Riyadh 11451, Saudi Arabia.

E-mail: F40M040@KSU.EDU.SA 